\title{
Feminizing Genitoplasty in Congenital Adrenal Hyperplasia: 20-year Experience of Regional Comprehensive Care
}

\author{
Katie Lynn Canalichio, " , Jennifer Ahn' ${ }^{1}$, Anne-Marie Elizabeth Amies Oelschlager², \\ Patricia Yvonne Fechner ${ }^{3}$, Elizabeth McCauley ${ }^{4}$, Linda Ramsdell ${ }^{5}$, Paul Merguerian ${ }^{1}$, \\ Margarett Shnorhavorian ${ }^{1}$
}

${ }^{1}$ Department of Pediatric Urology, Seattle Children's Hospital, Seattle, United States

${ }^{2}$ Department of Adolescent Gynecology, University of Washington, Seattle, United States

${ }^{3}$ Department of Pediatric Endocrinology, Seattle Children's Hospital, Seattle, United States

${ }^{4}$ Department of Behavioral Medicine, Seattle Children's Hospital, Seattle, United States

${ }^{5}$ Department of Genetics, Seattle Children's Hospital, Seattle, United States

Email address:

Katie.L.Canalichio@gmail.com (K. L. Canalichio)

${ }^{*}$ Corresponding author

\section{To cite this article:}

Katie Lynn Canalichio, Jennifer Ahn, Anne-Marie Elizabeth Amies Oelschlager, Patricia Yvonne Fechner, Elizabeth McCauley, Linda Ramsdell, Paul Merguerian, Margarett Shnorhavorian. Feminizing Genitoplasty in Congenital Adrenal Hyperplasia: 20-year Experience of Regional Comprehensive Care. International Journal of Clinical Urology. Vol. 4, No. 2, 2020, pp. 45-50. doi: 10.11648/j.ijcu.20200402.12

Received: June 10, 2020; Accepted: June 28, 2020; Published: July 13, 2020

\begin{abstract}
Background: There is need for data on long-term patient outcomes following feminizing genitoplasty (FG) in females with congenital adrenal hyperplasia $(\mathrm{CAH})$. We aim to describe postoperative and longitudinal psychosocial, endocrine, gynecologic, and urologic outcomes. Methods: We performed a retrospective review of past 20 years looking at females with CAH status post FG. Patients without follow-up were excluded. We captured demographic data, intraoperative details, 30-day complication rates, and longitudinal outcomes. Results: Twenty patients met inclusion criteria. Prader scores ranged from 3-5. Median age at surgery was 1.0 year (IQR 0.6, 1.3). Median follow-up was 4.5 years (IQR 0.7, 9.6). Genetic testing was performed in 15/20 and all had causative variants in the CYP21A2 gene. Thirty-day surgical complications occurred in 5/20 patients (Clavien I-IIIb). All patients over 3 years were toilet trained, with only 1 patient requiring surgery for urinary incontinence. In post-pubertal patients, $5 / 6$ had vaginal stenosis at median age 17.8 years (IQR 14.9, 21.1). Four patients received treatment for behavioral/psychiatric diagnoses. No patient had gender dysphoria. Conclusion: FG is safe as demonstrated by our low major complication rate. Our experience highlights the value of multidisciplinary care through adolescence and young adulthood to support long-term medical and psychosocial outcomes in females with CAH.
\end{abstract}

Keywords: Congenital Adrenal Hyperplasia, Feminizing Genitoplasty, Surgical Outcomes, Urinary Incontinence, Vaginal Stenosis

\section{Introduction}

Ambiguous genitalia occurs when anatomic, gonadal and/or chromosomal sex are in disagreement; it has been shown to occur in 1 in 1,000-4,500 live births [1]. Congenital adrenal hyperplasia $(\mathrm{CAH})$, the most common cause of ambiguous genitalia in the newborn female, is due to deficiencies in the enzymes responsible for cortisol synthesis.
The most common deficiency occurs with 21-hydroxylase and can be further classified as 1) salt-wasting 2) simple virilizing or 3) nonclassic forms [2]. In 46, XX females with $\mathrm{CAH}$, a spectrum of virilization of the external genitalia occurs in relation to the amount and timing of androgen exposure, with formation of typical proximal vagina, uterus, fallopian tubes, and ovaries $[1,2]$. The traditional approach to these patients was to perform feminizing genitoplasty in 
the first 3-6 months of life to form a separate urethral and vaginal opening from the urogenital sinus and to decrease the size of the clitoris, with the thought that this would minimize the functional and psychological impact on the child $[2,3]$. Recently, some have recommended delaying or avoiding surgery until age of patient consent [3]. Given the complex anatomic considerations and multifactorial nature of psychosexual development, the contemporary approach involves multidisciplinary teams with focus on both parents and patients with shared decision-making [1, 4, 5].

Limited data in the literature is available for long-term patient outcomes following feminizing genitoplasty in female patients with $\mathrm{CAH}$ [5]. In this study, we plan to describe urological, gynecological, endocrine, genetic and psychosocial long-term outcomes in this patient population at a single tertiary center. Our secondary aim is to describe postoperative complications within this cohort and identify risk factors for these. We hypothesize that early feminizing genitoplasty is safe in females with $\mathrm{CAH}$.

\section{Methods}

We obtained IRB approval at our institution. We retrospectively reviewed our institutional database from January 1999 through January 2019. We identified females with diagnosis of CAH based on ICD codes (ICD 10 - E25.0; ICD 9 - 255.2; ICD 8 - 255, 255.0, 255.9, 758, 758.1, 758.8, 758.9) who underwent surgical genitourinary reconstruction. Inclusion criteria were diagnosis of $\mathrm{CAH}$, female patient, and undergoing feminizing genitoplasty at our institution. Patients with no operative data or minimum of 30-day follow-up data were excluded. Data was housed in REDCap electronic database.

Demographic information, preoperative evaluations, intraoperative measurements, postoperative follow-up, and long-term outcomes were obtained from the electronic medical record. The operative reports, including nursing and anesthesia documentation, were reviewed for each case. We collected postoperative complications within a 30-day period by reviewing the chart for discharge documentation, emergency room visits, readmission notes, and clinic followup. We further defined any 30-day surgical complications based on the Clavien-Dindo classification system [6]. Descriptive statistics were employed to measure median and interquartile ranges (IQR) where appropriate.

Length of follow-up was calculated from date of surgery to last follow-up with our multidisciplinary clinic or an individual associated clinic (urology, gynecology, behavioral medicine, endocrinology, and genetics). From the respective subspecialty visits, we were able to glean information on longterm endocrine, urinary, vaginal and psychosocial outcomes. We recorded patient's involvement in our multidisciplinary clinic and what services were involved in preoperative and postoperative assessment based on specialty.

Urinary incontinence was defined by the International Children's Continence Society (ICCS) 2014 definition. Reported symptoms were broken down as continuous or intermittent in nature. Intermittent could be either daytime and/or nighttime [7]. We only assessed for incontinence in children $>3$ years-old based on their age-appropriateness to achieve toilet training. Recurrent urinary tract infections were defined by the common definition of two distinct documented infections in a six-month period or three in one year [8]. For purposes of this review, this was described in the medical record, either by direct access to the urine culture result or mention of urinary tract infection in the clinic notes.

Vaginal stenosis was reported based on the need for dilation, surgery and/or symptomatic presentation. Vaginal stenosis was defined as severe in those requiring vaginorrhaphy or mild in those that could be managed with vaginal dilation alone. We looked at the proportion of vaginal stenosis in the entire cohort, but also separately in patients who had achieved menarche or were post-pubertal.

Axis I disorders were made based on diagnostic criteria listed in the DSM-IV. Behavioral issues, not diagnosable as an Axis I disorder, were also gleaned from the medical record.

\section{Results}

Twenty-seven patients were identified. Seven patients were excluded because of missing intraoperative reports, having undergone initial feminizing genitoplasty at another institution, or missing the required minimum length of follow-up. Median age at surgery was 1.0 year (IQR 0.6, 1.3) with a median follow-up of 4.5 years (IQR 0.7, 9.6). Eighteen of the 20 patients were evaluated by a multidisciplinary care team, which included all patients seen in the past 10 years. The majority of patients were nonHispanic white $(13 / 20)$. The remainder were non-Hispanic black (1/20), Hispanic (5/20), or Asian (1/20). Educational level at last follow-up included non-school aged children $(8 / 20)$, elementary $(5 / 20)$, middle school $(1 / 20)$, high school $(5 / 20)$, and was not recorded in the remaining patient $(1 / 20)$. Nineteen $(95 \%)$ patients were diagnosed with classic 21 hydroxylase deficiency and the subtype was not recorded in one patient. Parent (s) of 16 of $20(80 \%)$ children received genetic counseling, which included all families seen in the past 10 years. Genetic testing was performed in 16 of 20 $(80 \%)$ patients and all had causative variants in the CYP21A2 gene identified. The remaining 4 (20\%) did not undergo genetic testing because of parental decline or were evaluated more than a decade ago without the benefit of the multidisciplinary clinic.

Sixteen of the 20 patients had separate pre-operative visits with the surgeon prior to undergoing feminizing genitoplasty - this was the standard for the past 10 years with $100 \%$ of patients in this timeframe. Six (30\%) patients underwent separate exam under anesthesia (EUA) prior to surgery. All patients had Prader scores ranging from 3 to 5 with a median Prader score of 4 (IQR 3,4) - this was directly mentioned in 12 patients on the physical exam findings. The urogenital confluence was recorded in 15 patients with a median length of $1.5 \mathrm{~cm}$ (IQR 1.0, 2.0). It was described as "low" in one additional patient. See Table 1 for additional intraoperative characteristics. 
Table 1. Patient Characteristics.

\begin{tabular}{lll}
\hline & Median & Interquartile range (IQR) \\
\hline Age at Surgery (years) & 1.0 & $0.6-1.3$ \\
Follow-up (years) & 4.5 & $0.7-9.6$ \\
Prader Score $\left({ }^{*} \mathrm{n}=12\right)$ & 4.0 & $3.0-4.0$ \\
Urogenital Confluence $(\mathrm{cm})\left({ }^{*} \mathrm{n}=15\right)$ & 1.5 & $1.0-2.0$ \\
Urethral Length $(\mathrm{cm})\left({ }^{*} \mathrm{n}=8\right)$ & 2.0 & $2.0-3.2$ \\
Vaginal Length $(\mathrm{cm})\left({ }^{*} \mathrm{n}=3\right)$ & 3.0 & --- \\
Stretched Clitoral Length $(\mathrm{cm})\left({ }^{\mathrm{n}}=14\right)$ & 2.8 & $2.0-3.5$ \\
\hline
\end{tabular}

$*_{n}$ (total number of patients this was recorded in).

The majority of cases were performed in the past 10 years $(\mathrm{n}=15,75 \%)$. Clitoroplasty was performed in $17(85 \%)$, with 16 specifically stating a nerve-sparing operation. Labioplasty was performed in $15(75 \%)$. Vaginoplasty was performed in $18(90 \%)$ : cut-back $(n=1)$ and flap vaginoplasty $(n=17)$. Partial urogenital mobilization was performed in 5 patients (25\%). No patient had total urogenital mobilization. See Table 2 for thirty-day surgical complications. These occurred in $5(25 \%)$ patients with only one requiring readmission and return to the operating room for surgical site infection (SSI) and wound separation (Clavien IIIb). Three (15\%) other patients experienced a combination of SSI and urinary tract infection (UTI) (Clavien II). The remaining patient had superficial skin separation (Clavien I).

Table 2. Patient Outcomes.

\begin{tabular}{ll}
\hline Outcomes & $\mathbf{n} / \mathbf{N}(\mathbf{\%})$ \\
\hline Genetic & \\
Genetic Counseling & $16 / 20(80 \%)$ \\
Genetic Testing & $15 / 20(75 \%)$ \\
Surgical 30-Day Complications & \\
Clavien I & $1 / 20(5 \%)$ \\
Clavien II & $3 / 20(15 \%)$ \\
Clavien IIIb & $1 / 20(5 \%)$ \\
Endocrine & \\
Perioperative Adrenal Crisis & $0 / 20(0 \%)$ \\
Psychosocial & \\
Behavioral Issues & $1 / 20(5 \%)$ \\
Axis 1 Diagnosis & $3 / 20(15 \%)$ \\
Gender Dysphoria & $0 / 20(0 \%)$ \\
Substance Abuse & $2 / 20(10 \%)$ \\
\hline
\end{tabular}

No patient suffered a perioperative adrenal crisis. Endocrine was consulted perioperatively for cases at our institution. Stress dose steroids were provided in the operating room to $16(80 \%)$ patients. Perioperative antibiotics were provided to $18(90 \%)$ patients. Six (30\%) patients had reached menarche, four $(67 \%)$ of whom reported irregular menses that were managed with oral contraceptives. Additional endocrine outcomes are listed in Figure 1 and reflect the level of endocrine control at last follow-up.

Two $(10 \%)$ patients had recurrent urinary tract infections. All patients over 3 years-old achieved toilet training $(n=16)$. Urinary incontinence was reported in $3(19 \%)$ patients symptoms were not mutually exclusive and were broken down into continuous $(n=2)$, daytime $(n=2)$ and enuresis $(n=2)$. Two patients had reassuring urodynamic studies with normal compliance, capacity and end-filling pressures and no demonstrated leak. Both had associated obesity and dysfunctional voiding that were managed with behavioral therapy alone. All but one have had resolution of urinary incontinence at last follow-up. See Table 3.

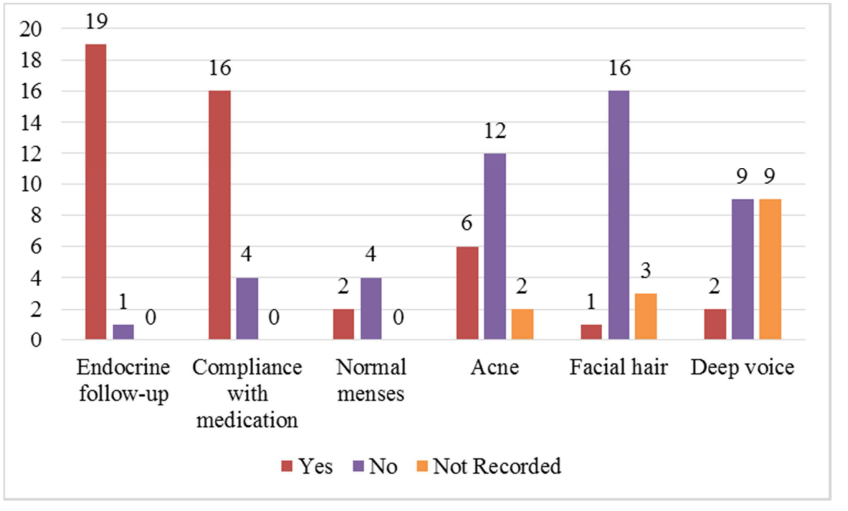

Figure 1. Endocrine Outcomes.

No patient required clean intermittent catheterization or anticholinergic use. The one patient who required surgery for urinary incontinence had concomitant vaginal stenosis and obesity. She was Prader 5 on initial exam with a high urogenital confluence and underwent a flap vaginoplasty at 5 months of age. Several years after her initial feminizing genitoplasty, she had an exam under anesthesia and was found to have an extremely hypospadiac urethra with florid leakage of urine and a separate vaginal orifice. She underwent buccal mucosa onlay vaginoplasty as well as urethroplasty to elongate the urethra by using the anterior vaginal wall mucosa. At last follow-up, she self-dilates her vagina without pain and no longer has urinary incontinence.

Overall, vaginal stenosis was seen in 5 of $20(25 \%)$ patients, with all cases presenting in post-pubertal patients. Of the 6 post-pubertal patients, 1 reported ability to use tampons, and 3 reported being sexually active. Of the 5 patients with vaginal stenosis, median age at diagnosis was 17.8 years (IQR 14.921.1). Two patients required vaginorrhaphy with buccal grafts (one of these included the patient described above in the urinary incontinence outcomes). One patient was planned at last follow-up to undergo vaginorrhaphy at the adult hospital. The remaining 2 patients were managed with self-vaginal dilation. Urethral length measured in the operating room was a 
median of $3 \mathrm{~cm}$ (IQR 2.8, 3.0). At last follow-up, all, except the one pending surgery at the outside hospital, were managed successfully with the optimal intervention (surgery or dilation). See Table 3.

Table 3. Urinary and Vaginal Outcomes.

\begin{tabular}{ll}
\hline Outcome & $\mathbf{n} / \mathbf{N}(\mathbf{\%})$ \\
\hline Urinary Outcomes & \\
Toilet Trained & $16 / 16(100 \%)^{*}$ \\
Incontinence (Any) & $3 / 16(19 \%)^{*}$ \\
Surgery for Incontinence & $1 / 16(6 \%)^{*}$ \\
Continent (at Last Follow-up) & $15 / 16(94 \%)^{*}$ \\
Recurrent UTIs & $2 / 20(10 \%)$ \\
Clean Intermittent Catheterization & $0 / 20(0 \%)$ \\
Urinary diversion & $0 / 20(0 \%)$ \\
Vaginal Outcomes & \\
Vaginal Stenosis (All Patients) & $5 / 20(25 \%)$ \\
Severe (Requiring Vaginorraphy) & $3 / 20(15 \%)$ \\
Mild (Self-Vaginal Dilation) & $2 / 20(10 \%)$ \\
Vaginal Stenosis (Post-pubertal) & $5 / 6(83 \%)^{* *}$ \\
Severe (Requiring Vaginorraphy) & $3 / 6(50 \%)^{* *}$ \\
Mild (Self-Vaginal Dilation) & $2 / 6(33 \%)^{* *}$ \\
Vaginal Stenosis (at Last Follow-up) & $1 / 6(17 \%)^{* *}$ \\
Sexually Active & $3 / 6(50 \%)$ \\
Obstructed Menses & $0 / 6(0 \%)$ \\
\hline
\end{tabular}

*continence outcomes reported in patients $>3$ years $(n=16)$.

**vaginal outcomes reported in post-pubertal patients $(n=6)$.

Twelve $(60 \%)$ patients had a dedicated follow-up visit with a behavioral therapist. Four $(20 \%)$ patients had behavioral issues identified and of these patients, 3 went on to have Axis 1 psychiatric diagnoses - depressive disorder NOS $(n=2)$ and adjustment disorder $(n=1)$. Behavioral issues included school-related difficulties, childhood aggression, and suicidal ideation. The 2 patients with depressive disorder also had history of substance abuse. No patient reported gender dysphoria at last follow-up. Differences in sexual attraction were reported in 3 of 20 patients (15\%): same-sex attraction $(n=2)$ and bisexual attraction $(n=1)$.

\section{Discussion}

There are no randomized controlled trials evaluating the best age of surgery for feminizing genitoplasty in CAH. Most available literature involves retrospective reviews [9]. Purported benefits of early surgery include preventing known issues such as urinary tract infections and hydrocolpos, minimizing perceived stigma, avoiding potential psychological trauma of genital surgery at older ages, and the positive maternal estrogen effect on the tissues [9, 10]. Known complications following feminizing genitoplasty include vaginal stenosis, scarring, loss of sexual function, urinary incontinence, fistula, and diminished clitoral sensitivity [9].

In our study, the overall 30-day surgical complication rate was $25 \%$, but the majority $(80 \%)$ of these were minor (Clavien I-II). These included urinary tract infection, minor surgical site infection and wound separation. Only one patient had a major complication that required takeback to the operating room for surgical site infection and wound separation. There were no reported fistulae and no patient suffered a perioperative adrenal crisis; endocrine was consulted in the perioperative period at our institution and appropriate stress dosing was provided in the operating room for the majority of cases. This data was comparable to the previous available literature. Dangle et al. performed a retrospective review to identify surgical complications after early feminzing genitoplasty. The authors identified 26 patients that were operated on at an average age of 17 months, with average follow-up of 72 months [10]. All patients underwent single-stage feminizing genitoplasty with clitoroplasty, labioplasty, and vaginoplasty with either partial $(n=9)$ or total $(n=14)$ urogenital mobilization. The overall complication rate was $27 \%$ - including dysuria, dehiscence/wound separation, and UTI - with only $7.6 \%$ requiring surgical revision with subsequent Clavien IIIb grade [10].

In this study, all 16 patients who were older than 3 years of age were able to be toilet trained. There was a high rate of urinary incontinence (19\%) in this cohort which was managed conservatively, except for in one $(6 \%)$. This patient, who required surgery, had highly virilized external genitalia with a high urogenital confluence, and underwent flap vaginoplasty as an infant which did not alleviate the reflux of urine into the vagina. Only one $(6 \%)$ of the patients remains symptomatic. This patient has a high BMI with evidence of vaginal voiding, and she has since embarked on behavioral techniques to help control this.

Stites et al. performed a retrospective review looking at urinary outcomes following vaginoplasty in CAH [11]. Twenty-four patients were identified, median age of surgery was 8 months, with a follow-up of 6 years, with only $62.5 \%$ achieving potty training without issue [11]. Dangle et al. found that urinary continence was attained in all (100\%) toilet-trained girls [10]. In our study, all but one patient were dry at the time of last follow-up (94\%). This highlights that there exists high success with treatment of urinary incontinence in these patients.

Vaginal outcomes were reported in our 6 post-pubertal patients. There was a high rate of vaginal stenosis within this group, with $50 \%$ having severe vaginal stenosis and $33 \%$ having mild vaginal stenosis. Of the patients that underwent surgery or self-vaginal dilation, all did well with selected management. When taken into context of the entire cohort, only $3 / 20(15 \%)$ had severe stenosis, as there were no reports of early vaginal stenosis. One patient reported tampon use (17\%) and 3 patients reported sexual activity (50\%). Our study did not address adult outcomes, so reproductive measures and additional sexual functioning outcomes were not assessed.

In 2018, Almasri et al. performed a systematic review and meta-analysis as part of guideline development [12]. The authors summarized 29 observational studies, the majority were retrospective and not comparative, which included 1,178 patients who underwent feminizing genitoplasty. There 
was high level of heterogeneity. The mean age at surgery was 2.7 years with average follow-up of 10.3 years. The most common complication reported was vaginal stenosis in $27 \%$ (spanning 14 of the articles). Other complications were reported with less frequency: 6\% UTI, 4\% urinary incontinence and $2 \%$ fistulae [12].

In our study no patients reported gender dysphoria. Existing literature has shown increased differences in sexual attraction among women with $\mathrm{CAH}$ [13], and this was reported in $3(15 \%)$ of the patients in our study. Of the four patients who had psychiatric or behavioral issues, three of them had same sex or bisexual attraction. Two of the patients who had depression, also had a history of substance abuse. Previous work describes good psychosocial outcomes in females with $\mathrm{CAH}$ with adjustment similar to the general population [14]. The expectation is for only a transient adjustment process as seen with other children with chronic illnesses; however, this was restricted to girls with early initiation of appropriate medical management [14]. These findings highlights the importance of close follow-up, multidisciplinary care, and an inclusive environment for patients to discuss sensitive topics.

Almasri et al. reviewed sexual outcomes in adult women, and despite $88.5 \%$ being sexually active, the mean Female Sexual Function Index (FSFI) score indicated sexual dysfunction with most women reporting some level of impairment [12]. Additionally, $88.7 \%$ patients reported female gender identity and $76.2 \%$ had heterosexual orientation. Strengths of this study included a rigorous design, summarizing the available outcomes on sexual health, but with obvious limitations of the inherent heterogeneous population and low quality evidence of the individual studies [12].

There has been a recent update with the $2018 \mathrm{CAH}$ Guidelines that were created in conjunction with a patient advocacy group, focusing on a comprehensive care model [9]. There has been a shift in our institution towards all patients being seen in a multidisciplinary clinic setting and the majority of cases of feminizing genitoplasty being performed in the past 10 years.

Limitations to our study include the retrospective nature and small patient population. When performing this chart review, we were unable to capture all desired data points and this became increasingly more difficult for cases that took place more than 10 years ago. In addition, we may be underestimating the true incidence of vaginal and psychiatric outcomes, as patients transitioning out of our pediatric institution may be lost to follow-up. In the past, not every patient was reflexively seen by the behavioral therapist, but this is a mandatory part of our evaluation now. Another limitation was that the majority of patients were under 2 years-old at age of surgery, so there are no comparisons available for those that chose late surgery. Finally, most of our patients are pre-pubertal, thus limiting report of longterm post-pubertal outcomes.

Strengths in our study include the robust multidisciplinary team exposure at our institution that is able to capture the genetic, endocrine, behavioral, vaginal and urinary outcomes in $\mathrm{CAH}$ patients. The detailed genetic and endocrine experience currently employed is key in providing families and patients with genetic counseling and optimal medical management of the underlying disease. The support provided from behavioral medicine is paramount to the functioning of these children, adolescents and families, and can help towards identifying those at risk. Additionally, these outcomes can be used to provide families with counseling material when presenting for care.

\section{Conclusion}

Our data supports excellent urological, gynecological, endocrine, genetic and psychosocial long-term outcomes when patients are cared for in a multidisciplinary team that can satisfactorily address expected outcomes. Feminizing genitoplasty is safe as evidenced by our low major complication rates. However, an overall $25 \%$ complication rate is noteworthy and continued follow-up is needed to help manage expected outcomes: vaginal stenosis, psychiatric/behavioral issues, and urinary incontinence. Long-term multidisciplinary follow-up, especially in adolescence, is critical for these patients. With our current model in place, we have been able to successfully manage these outcomes. Future directions include continued longterm follow-up and comparing outcomes to patients who choose not to undergo feminizing genitoplasty.

\section{Recommendations}

We recommend that patients with $\mathrm{CAH}$ be seen at comprehensive care centers to promote optimal outcomes. This will allow for optimal endocrine control, close followup with a behavioral medicine, appropriate counseling of families with female newborns with $\mathrm{CAH}$, correct choice of surgical technique, and expectant versus reactionary management of known issues for these patients.

\section{Author Contributions}

All authors were involved in conception, data analysis and interpretation, critical revision, and final approval of this version of the work. The first author performed the data collection and drafting of this article.

\section{Conflict of Interest Statement}

None.

\section{Ethics Approval}

Institutional approval obtained.

\section{Acknowledgements}

None. 


\section{References}

[1] Baskin LS. Restoring normal anatomy in female patients with atypical genitalia. Semin Perinatol. 2017; 41 (4): 227-31. doi: 10.1053/j.semperi.2017.03.011. PubMed PMID: 28535944.

[2] Diamond DA YR. Disorders of Sexual Development: Etiology, Evaluation, and Medical Management. In: Wein AJ KL, Partin AW, Peters CA, editor. Campbell-Walsh Urology. 4. Philadelphia, PA: Elsevier Saunders; 2016. p. 3469-97.

[3] Szymanski KM, Whittam B, Kaefer M, Frady H, Casey JT, Tran VT, et al. Parental decisional regret and views about optimal timing of female genital restoration surgery in congenital adrenal hyperplasia. J Pediatr Urol. 2018; 14 (2): 156 e1- e7. doi: 10.1016/j.jpurol.2017.11.012. PubMed PMID: 29330019.

[4] Auchus RJ, Witchel SF, Leight KR, Aisenberg J, Azziz R, Bachega TA, et al. Guidelines for the Development of Comprehensive Care Centers for Congenital Adrenal Hyperplasia: Guidance from the CARES Foundation Initiative. Int J Pediatr Endocrinol. 2010; 2010: 275213. doi: 10.1155/2010/275213. PubMed PMID: 21274448; PubMed Central PMCID: PMCPMC3025377.

[5] Speiser PW, Azziz R, Baskin LS, Ghizzoni L, Hensle TW, Merke DP, et al. A Summary of the Endocrine Society Clinical Practice Guidelines on Congenital Adrenal Hyperplasia due to Steroid 21-Hydroxylase Deficiency. Int J Pediatr Endocrinol. 2010; 2010: 494173. doi: 10.1155/2010/494173. PubMed PMID: 20981249; PubMed Central PMCID: PMCPMC2963799.

[6] Dindo D, Demartines N, Clavien PA. Classification of surgical complications: a new proposal with evaluation in a cohort of 6336 patients and results of a survey. Ann Surg. 2004; 240 (2): 205-13. doi: 10.1097/01.sla.0000133083.54934.ae. PubMed PMID: 15273542; PubMed Central PMCID: PMCPMC1360123.

[7] Austin PF, Bauer SB, Bower W, Chase J, Franco I, Hoebeke P, et al. The standardization of terminology of lower urinary tract function in children and adolescents: Update report from the standardization committee of the International Children's
Continence Society. Neurourol Urodyn. 2016; 35 (4): 471-81. doi: 10.1002/nau.22751. PubMed PMID: 25772695.

[8] Anger J, Lee U, Ackerman AL, Chou R, Chughtai B, Clemens $\mathrm{JQ}$, et al. Recurrent Uncomplicated Urinary Tract Infections in Women: AUA/CUA/SUFU Guideline. J Urol. 2019; 202 (2): 282-9. doi: 10.1097/JU.0000000000000296.PubMedPMID: 31042112 .

[9] Speiser PW, Arlt W, Auchus RJ, Baskin LS, Conway GS, Merke DP, et al. Congenital Adrenal Hyperplasia Due to Steroid 21-Hydroxylase Deficiency: An Endocrine Society Clinical Practice Guideline. J Clin Endocrinol Metab. 2018; 103 (11): 4043-88. doi: 10.1210/jc.2018-01865. PubMed PMID: 30272171.

[10] Dangle PP, Lee A, Chaudhry R, Schneck FX. Surgical Complications Following Early Genitourinary Reconstructive Surgery for Congenital Adrenal Hyperplasia-Interim Analysis at 6 Years. Urology. 2017; 101: 111-5. doi: 10.1016/j.urology.2016.11.027. PubMed PMID: 27889492.

[11] Stites J, Bernabe KJ, Galan D, Felsen D, Poppas DP. Urinary continence outcomes following vaginoplasty in patients with congenital adrenal hyperplasia. J Pediatr Urol. 2017; 13 (1): 38 e1- e7. doi: 10.1016/j.jpurol.2016.10.012. PubMed PMID: 27932079 .

[12] Almasri J, Zaiem F, Rodriguez-Gutierrez R, Tamhane SU, Iqbal AM, Prokop LJ, et al. Genital Reconstructive Surgery in Females With Congenital Adrenal Hyperplasia: A Systematic Review and Meta-Analysis. J Clin Endocrinol Metab. 2018; 103 (11): 4089-96. doi: 10.1210/jc.2018-01863. PubMed PMID: 30272250.

[13] Meyer-Bahlburg HF, Dolezal C, Baker SW, New MI. Sexual orientation in women with classical or non-classical congenital adrenal hyperplasia as a function of degree of prenatal androgen excess. Arch Sex Behav. 2008; 37 (1): 8599. doi: 10.1007/s10508-007-9265-1. PubMed PMID: 18157628.

[14] Berenbaum SA, Korman Bryk K, Duck SC, Resnick SM. Psychological adjustment in children and adults with congenital adrenal hyperplasia. J Pediatr. 2004; 144 (6): 7416. doi: 10.1016/j.jpeds.2004.03.037. PubMed PMID: 15192620 . 